\title{
Fratura epifisiolise da extremidade proximal do úmero com luxação intratorácica: relato de caso*
}

\author{
Epiphysiolysis fracture of the proximal end of the \\ humerus with intrathoracic dislocation: a case report
}

Jaime Guiotti Filho', Antônio Carlos Wall Borges², Marcelo de Carvalho Leite ${ }^{3}$

\section{RESUMO}

A fratura da extremidade proximal do úmero com luxação intratorácica foi relatada em 1949 por West, em que a fratura era, somente, do tubérculo maior. Desde então, poucos casos foram relatados na literatura, a maioria constituída por pessoas idosas, prevalecendo como indicação terapêutica artroplastia parcial. Os autores relatam o caso de um adolescente de 14 anos de idade, sexo masculino, que apresentou fratura epifisiolise da extremidade proximal do úmero com luxação intratorácica em decorrência de acidente ciclístico e que foi submetido a tratamento cirúrgico com redução, osteossíntese e reinserção do manguito rotador. A recuperação da cabeça do úmero totalmente desvitalizada e o acompanhamento do processo de necrose e revas-

* Trabalho realizado no Setor de Ombro e Cotovelo do Serviço de Residência Médica do Instituto Ortopédico de Goiânia (IOG), Goiânia (GO), Brasil.

1. Médico Ortopedista; Coordenador do Setor de Ombro e Cotovelo do Serviço de Residência Médica do Instituto Ortopédico de Goiânia.

2. Médico Ortopedista; Assistente do Setor de Ombro e Cotovelo do Serviço de Residência Médica do Instituto Ortopédico de Goiânia.

3. Médico estagiário do Setor de Ombro e Cotovelo do Serviço de Residência Médica do Instituto Ortopédico de Goiânia.

Endereço para correspondência: Jaime Guiotti Filho, Rua T-27, esquina com T-49, no 819, Setor Bueno - 74210-030 - Goiânia (GO), Brasil. Tel.: (62) 3252-5000. E-mail: jguiotti@uol.com.br Recebido em 7/8/07. Aprovado para publicação em 24/1/08. Copyright RBO2008 cularização durante seis anos, em paciente adolescente, parece não terem sido previamente relatados.

Descritores - Epifisiolise; Necrose asséptica do osso; Relato de caso

\section{ABSTRACT}

Fracture of the proximal end of the humerus with intrathoracic dislocation was reported in 1949 by West, and the fracture was only a fracture of the greater tubercle. Few cases have since been published, and most of them in elderly individuals, partial arthroplasty prevailing as the therapy indication. The authors report the case of a 14 year old boy who presented with an epiphysiolysis fracture of the proximal end of the humerus with intrathoracic dislocation resulting from a bicycle accident. The boy was submitted to surgical treatment with reduction, osteosynthesis, and reinsertion of the rotator cuff. The totally devitalized humeral head recovery and the monitoring of the necrosis and revascularization process for a period of six years in a teenager patient seems to have never been reported before.

Keywords - Epiphysiolysis; Aseptic necrosis of the bone; Case report

\section{INTRODUÇÃO}

A fratura luxação intratorácica da extremidade proximal do úmero é extremamente $\operatorname{rara}^{(1)}$. Essa lesão tem sido relatada em pacientes idosos, após queda com a 
extremidade superior em abdução ${ }^{(2-3)}$, e em jovens, após trauma de alta energia ${ }^{(4-5)}$. Encontramos oito casos relatados na literatura, sendo dois de luxação com fratura do tubérculo maior, em que o paciente mais jovem tinha 14 anos, e, nos outros seis, a cabeça do úmero estava solta sem nenhuma continuidade com a diáfise do úmero, cujo tratamento foi, na sua maioria, artroplastia parcial. O caso relatado mais recentemente é o de um paciente do sexo masculino, 27 anos de idade, em que o fragmento da cabeça foi recuperado através de incisão deltopeitoral estendida, permitindo a execução de osteossíntese com resultado satisfatório após um ano de seguimento.

\section{RELATO DO CASO}

Em junho de 2001, um adolescente de 14 anos de idade, do sexo masculino, obeso, destro dominante, sofreu queda de bicicleta, apresentando trauma em membro superior direito, tórax e face, sem perda de consciência. Atendido na Emergência do Instituto Ortopédico de Goiânia, o paciente queixava-se de dor intensa no ombro e hemitórax direito e deambulava com o membro superior direito junto ao corpo. Ao exame físico apresentava escoriações em face, aumento de volume no ombro direito, sem déficit sensitivo e pulsos periféricos palpáveis.

O estudo por imagens revelou fratura epifisiolise da extremidade proximal do úmero com luxação intratorácica do fragmento da cabeça do úmero (figura 1) e fratura do segundo arco costal do lado direito. A tomografia computadorizada confirmou a presença da cabeça do úmero na cavidade torácica e hemopneumotórax (figura 2).

O paciente foi encaminhado imediatamente ao centro cirúrgico e submetido à toracotomia no terceiro espaço intercostal. Foram identificados na cavidade torácica: hemotórax, laceração do lobo superior do pulmão direito e a cabeça do úmero solta e não aderida a qualquer tecido. A cabeça do úmero foi então retirada através do acesso da toracotomia e mantida em um recipiente com soro fisiológico, enquanto eram realizadas a pneumorrafia do lobo superior do pulmão e drenagem torácica.

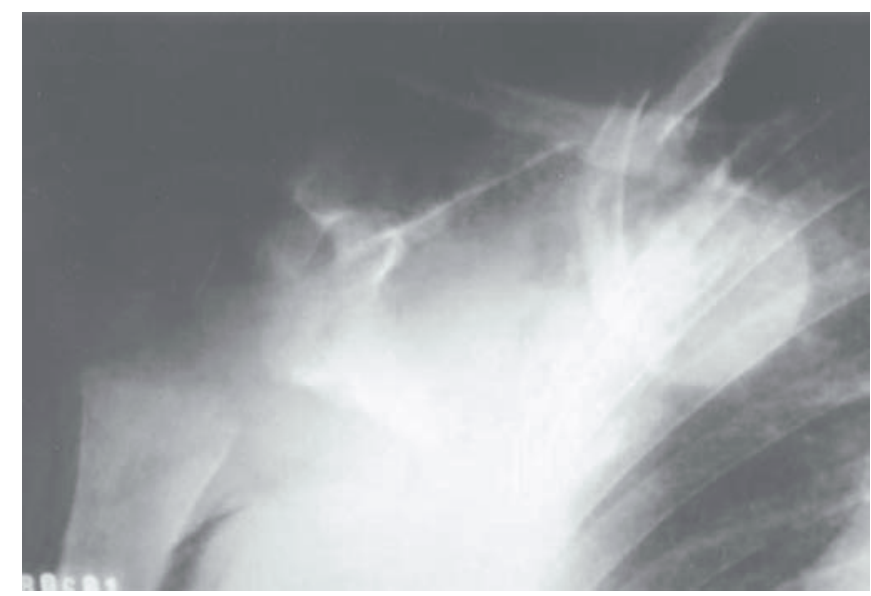

Figura 1 - Radiografia inicial demonstrando fratura da extremidade proximal do úmero com fragmento da cabeça dentro da cavidade torácica

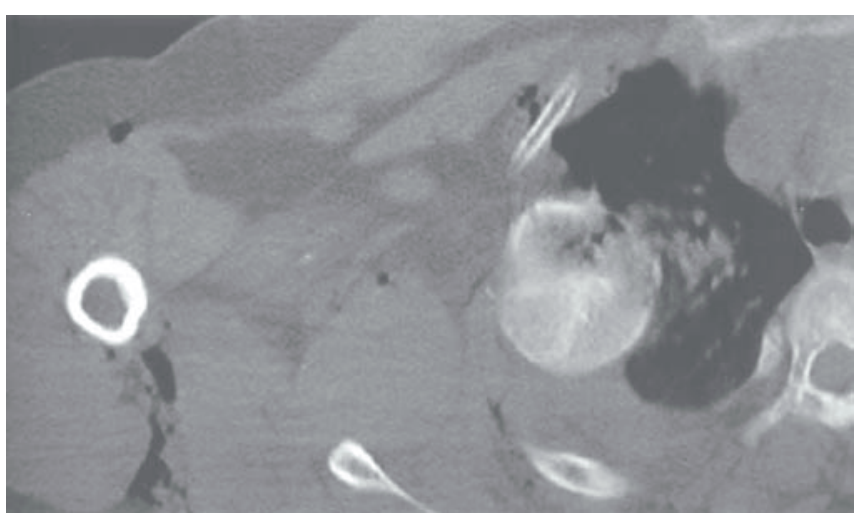

Figura 2 - Tomografia computadorizada evidenciando o fragmento da cabeça do úmero dentro da cavidade torácica e hemopneumotórax

Através de incisão deltopeitoral e acesso à extremidade proximal do úmero direito, foi encontrada lesão longitudinal do tendão do subescapular, sem a presença do tubérculo menor e fragmentos do tubérculo maior com os tendões do supra-espinhal, infra-espinhal e redondo menor. A cabeça longa do bíceps estava intacta e havia lesão labral na borda anterior da cavidade glenoidal. $\mathrm{O}$ fragmento da cabeça foi reduzido anatomicamente na diáfise do úmero e fixado por meio de dois pinos lisos (figura 3). Feita reinserção labral por pontos transósseos e em seguida realizada a redução da cabeça do úmero na cavidade glenoidal. O subescapular foi reconstruído e reinserido no tubérculo menor, que permanecia preso à cabeça do úmero; o tubérculo 


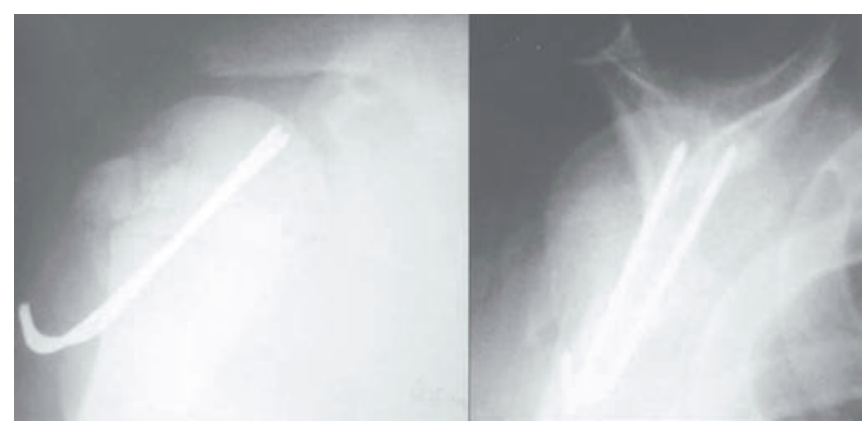

Figura 3 - Radiografia demonstrando a cabeça do úmero fixada com dois pinos lisos e redução da luxação após reinserção do manguito rotador

maior, reimplantado em sua topografia juntamente com suas inserções tendinosas. Toda essa reconstrução foi feita por pontos transósseos com a utilização de fios inabsorvíveis no 5 . A cabeça longa do bíceps foi reduzida no sulco intertubercular e o espaço dos rotadores foi fechado.

No pós-operatório, o paciente foi mantido em tipóia simples permanecendo internado por cinco dias. Os pontos foram retirados com duas semanas, os exercícios passivos iniciados com seis semanas e ativo-assistidos após três meses. Com quatro meses, o paciente apresentava-se sem dor, com elevação ativa de $45^{\circ}$, rotação externa de $45^{\circ}$ e rotação interna em região sacra. Nessa ocasião, as radiografias não mostravam sinais de necrose, a redução estava mantida e havia sinais de presença de calo ósseo. No sexto mês, foi retirado o material de síntese e era possível visualizar sinais de necrose incipientes. No sétimo mês, todos os movimentos do ombro direito estavam iguais aos do ombro esquerdo (elevação - $180^{\circ}$; RE - $90^{\circ}$ e RI - T-9), porém, o paciente queixava-se de diminuição de força sem comprometimento da função.

Com um ano, as radiografias apresentavam sinais de deformação e reabsorção da cabeça do úmero. Foi solicitada ressonância magnética e confirmada a presença de necrose. $\mathrm{O}$ processo de necrose avascular permaneceu em atividade até o terceiro ano, quando foi notada revascularização total, mas a cabeça do úmero deformada sem, entretanto, comprometer a congruência articular, permanecendo o mesmo padrão radiográfico com seis anos de avaliação (figura 4). $\mathrm{Na}$

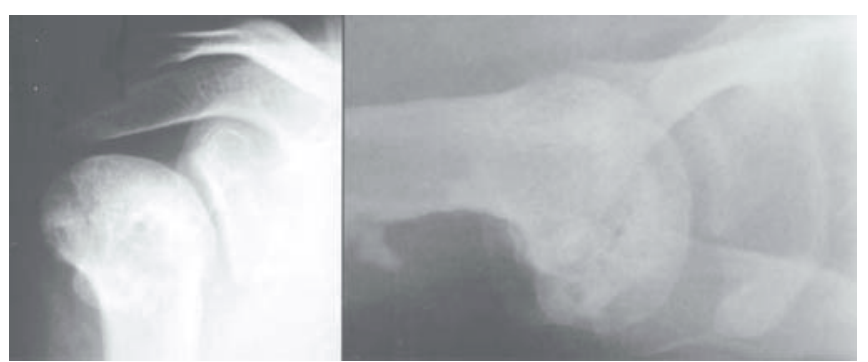

Figura 4 - Radiografia com seis anos de evolução evidenciando deformação da cabeça do úmero após processo de necrose e revascularização

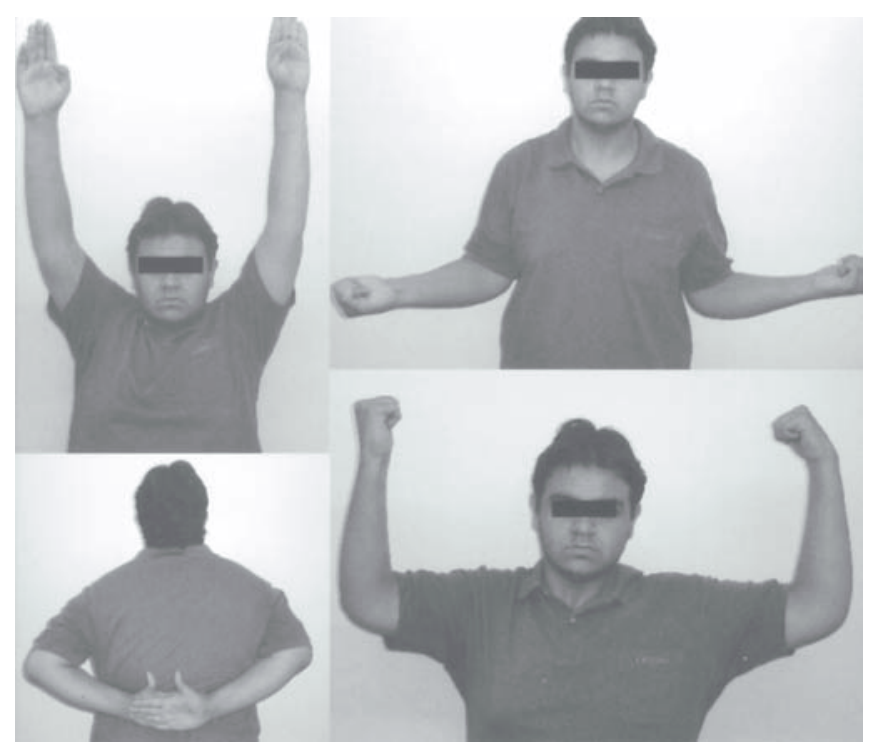

Figura 5 - Resultado funcional após seis anos de evolução

ocasião, os movimentos e a força do ombro direito estavam iguais aos do ombro contralateral. Durante todo o tempo de evolução, a partir do sétimo mês, a função do ombro comprometido esteve igual à do ombro normal e o paciente não teve queixa de dor, retomando suas atividades habituais sem restrições (figura 5).

\section{DISCUSSÃO}

A fratura luxação da extremidade proximal do úmero é uma lesão rara com consequiências potenciais devastadoras, particularmente em pacientes jovens e ativos. O padrão da lesão foi originalmente descrito por West, quando relatou o caso de um paciente vítima de trauma de alta energia em que a cabeça do úmero se 
manteve presa à porção proximal do osso e foi reduzida sob tração, sendo a fratura luxação do tubérculo maior tratada conservadoramente, evoluindo com limitação da abdução do ombro ${ }^{(4)}$. Simpson et al relataram um caso semelhante, no qual a redução foi facilitada pela manipulação digital através da incisão da toracotomia seguida de fixação interna do tubérculo maior e reparo do manguito rotador, tendo o paciente retorno completo de suas funções após fisioterapia prolongada ${ }^{(5)}$. Nos dois casos citados, a única lesão foi a fratura do tubérculo maior. Há outros seis casos descritos na literatura de fratura luxação intratorácica em que o fragmento da cabeça do úmero não estava preso à diáfise, sendo o mais recente o de um paciente de 27 anos de idade, o mais jovem já descrito ${ }^{(2-3,7-10)}$. Wirth et al relataram um caso de fratura da extremidade proximal do úmero com luxação retroperitoneal em uma paciente de 28 anos, tendo o fragmento da cabeça do úmero sido recuperado e fixado com dois parafusos; $\mathrm{o}$ seguimento após seis anos mostrou que houve necrose da cabeça do úmero, com evolução para acentuada deformidade, conseqüente artrose glenoumeral e redução da função do ombro ${ }^{(6)}$. Glessner descreveu um caso em que o fragmento da cabeça do úmero estava solto e foi cirurgicamente removido e descartado, sendo o remanescente do manguito rotador suturado na diáfise do úmero; o resultado foi satisfatório para um paciente de demanda funcional baixa ${ }^{(2)}$. Patel et al, em caso semelhante, optaram por deixar o fragmento da cabeça do úmero dentro da cavidade torácica e o seguimento radiográfico mostrou a fusão do tubérculo maior e diáfise do úmero na escápula; o resultado funcional foi relatado como satisfatório, pois se tratava de um paciente de baixa demanda funcional ${ }^{(3)}$. Eberson et al relataram caso de paciente do sexo masculino, com 64 anos de idade, que caiu de uma altura de $5 \mathrm{~m}$ sofrendo fratura luxação intratorácica contralateral; o fragmento da cabeça do úmero foi retirado da cavidade torácica por acesso guiado por toracoscopia, sendo realizada hemiartroplastia. $\mathrm{O}$ paciente teve resultado funcional satisfatório, apesar da neuropatia do plexo braquial que desenvolveu ${ }^{(7)}$. Harman et al descreveram caso de paciente de 56 anos de idade, sexo feminino, que apresentava fratura luxação intratoráci- ca por trauma de alta energia, a qual foi tratada por hemiartroplastia após retirada da cabeça do úmero por toracotomia ${ }^{(8)}$.

A vascularização da cabeça do úmero é sabidamente prejudicada em traumas de alta energia com fratura em vários fragmentos. O suprimento arterial primário da extremidade proximal do úmero é feito por um ramo da artéria circunflexa umeral anterior e a contribuição vascular de menor valor surge da artéria circunflexa umeral posterior e vasos, que entram através das aderências dos tecidos moles do manguito rotador ${ }^{(9)}$. Tem sido proposto que, durante a redução aberta e fixação interna, a proteção dos tecidos moles e suas aderências pode preservar algum fluxo para a cabeça do úmero ${ }^{(10)}$.

O relato mais recente de fratura luxação intratorácica foi feito por Anderson et al, em que o acesso para alcançar o fragmento da cabeça do úmero foi realizado através de incisão deltopeitoral estendida, permitindo manter a preservação de tecidos remanescentes do manguito rotador; esses tecidos foram suficientes para preservar a irrigação do fragmento da cabeça umeral, reinserida por meio de osteossíntese, com placa de estabilidade angular para extremidade proximal do úmero (Synthes $L C P^{\circledR}$ ) e sutura em banda de tensão fixando os tubérculos, obtendo consolidação sem necrose com um ano de evolução(11). Os autores chamam a atenção para o acesso deltopeitoral estendido que, embora tecnicamente difícil, pode permitir a preservação da irrigação através dos vasos oriundos do manguito rotador, os quais podem estar mantidos na cabeça do úmero na hora da lesão. Além do mais, esse acesso permite inspeção direta e reparo de qualquer lesão neurovascular que poderia ocorrer no momento da luxação intratorácica. Anderson et al acreditam que, nesse caso, a perfusão da cabeça do úmero foi mantida através da preservação dos tecidos moles aderidos do subescapular, até a reconstituição da perfusão interóssea ocorrer no período pós-operatório ${ }^{(11)}$.

Em nosso caso, o fragmento da cabeça do úmero foi encontrado sem aderência dos tecidos moles que pudessem manter a irrigação da mesma. O manguito rotador permaneceu junto à diáfise do úmero e o subescapular encontrava-se desprovido de tecido ósseo e com ruptura no sentido longitudinal. Mesmo que fos- 
se realizado o acesso deltopeitoral estendido, como preconizam Anderson et al, não seria possível preservar qualquer irrigação do fragmento da cabeça do úmero. Consideramos a possibilidade da revascularização da cabeça do úmero, tanto pelos vasos vindos do manguito rotador, quanto pelos vasos circunflexos, e optamos pela redução e osteossíntese do descolamento epifisário, seguindo-se reinserção do manguito rotador. Estávamos conscientes do risco de necrose, porém, por se tratar de paciente jovem e como a articulação do ombro não sofre carga direta, acreditamos que seria possível manter a congruência articular, mesmo com o surgimento da deformação da cabeça do úmero durante o processo de necrose até a revascularização fu-

\section{REFERÊNCIAS}

1. Rockwood CA Jr, Thomas, SC, Matsen FA. Subluxation and dislocations about the glenohumeral joint. In: Rockwood CA Jr, DP Green, RW Bucholz, eds. Rockwood and Green's fractures in adults, 3rd edition, Volume 1. Philadelphia, PA: JB Lippincott; 1991. p. 1042-4.

2. Glessner JR Jr. Intrathoracic dislocation of the humeral head. J Bone Joint Surg Am. 1961;43:428-30.

3. Patel MR, Pardee ML, Singerman RC. Intrathoracic dislocation of the head of the humerus. J Bone Joint Surg Am. 1963;45: 1712-4.

4. West EF. Intrathoracic dislocation of the humerus. J Bone Joint Surg Br. 1949;31(1):61-2.

5. Simpson NS, Schwappach JR, Toby EB. Fracture-dislocation of the humerus with intrathoracic displacement of the humeral head. A case report. J Bone Joint Surg Am. 1998;80(6):889-91.

6. Wirth MA, Jensen KL, Agarwal A, Curtis RJ, Rockwood Jr CA. Fracture-dislocation of the proximal part of the humerus with tura e definitiva. Assim, nossa expectativa foi alcançada e o paciente mantém a função normal do ombro, sem dor, embora apresente deformação da cabeça do úmero. É questionável, difícil e pouco provável, em casos como este, a manutenção da irrigação no fragmento da cabeça do úmero mesmo utilizando acesso deltopeitoral estendido.

O seguimento de seis anos do caso aqui relatado foi de fundamental importância para a avaliação final, diferenciando do caso descrito por Anderson et al, cujo tempo de seguimento foi de um ano. Enfatizamos que, a necrose asséptica da cabeça do úmero, no caso por nós relatado, tornou-se evidente a partir de um ano de evolução.

retroperitoneal displacement of the humeral head. A case report. J Bone and Joint Surg Am. 1997;79(5):763-6.

7. Eberson $\mathrm{CP}, \mathrm{Ng} \mathrm{T}$, Green A. Contralateral intrathoracic displacement of the humeral head. A case report. J Bone Joint Surg Am. 2000;82(1):105-8.

8. Harman BD, Miller NG, Probe RA. Intrathoracic humeral head fracture-dislocation. J Orthop Trauma. 2004;18(2):112-5.

9. Laing PG. The arterial supply of the adult humerus. J Bone Joint Surg Am. 1956;38(5):1105-16.

10. Wijgman AJ, Roolker W, Patt TW, Raaymakers EL, Marti RK. Open reduction and internal fixation of three and four-part fractures of the proximal part of the humerus. J Bone Joint Surg Am. 2002;84(11):1919-25.

11. Anderson M, Rose P, Jacofsky DJ, Torchia ME, Dahm DL. Intrathoracic fracture-dislocation of the proximal humerus: a case report and report of a new surgical technique. J Trauma. 2007;63(4):920-3. 